\title{
Large Energy Transfer Distance to a plane of Gold Nanoparticles
}

\author{
Xia Zhang ${ }^{1}$, Valerie A. Gerard ${ }^{2}$, Yurii K. Gun'ko ${ }^{2}$, Vladimir Lesnyak ${ }^{3}$, Nikolai Gaponik ${ }^{3}$, Andrei S. \\ Susha ${ }^{4}$, Andrey L. Rogach ${ }^{4}$ and A. Louise Bradley ${ }^{*}$, \\ ${ }^{I}$ Semiconductor Photonics Group, School of Physics, Trinity College Dublin, Dublin 2, Ireland \\ ${ }^{2}$ School of Chemistry, Trinity College Dublin, Dublin 2, Ireland \\ ${ }^{3}$ Physical Chemistry, TU Dresden, Bergstr. 66b, 01062 Dresden, Germany \\ ${ }^{4}$ Department of Physics and Materials Science \& Centre for Functional Photonics (CFP), City University of \\ Hong Kong, Hong Kong, China \\ "Tel: +353 (0)1 896 3595, Fax: +353 (0)1 67 11759, e-mail: bradlel@tcd.ie
}

\begin{abstract}
The quenching of emission in proximity to metallic surfaces via non-radiative energy transfer is studied for sensing applications. It can also be used for the measurement of distances on the nanoscale. We report on energy transfer from a plane of CdTe quantum dots (QDs) plane to a plane of gold (Au) nanoparticles. Both photoluminescence (PL) and luminescence lifetime measurements demonstrate that energy transfer efficiency not only depends on plane separation but also Au nanoparticle concentration. Energy transfer to the plane of metal nanoparticles can be considered within the standard Förster resonant energy transfer (FRET) model or the nano-metal surface energy transfer (NSET) model. It is found that both dependences are well described within the model of FRET which shows a $1 / \mathrm{d}^{4}$ distance dependence and a $1 / \mathrm{C}_{\mathrm{Au}} \mathrm{Au}$ concentration dependence. However, surprisingly large Förster radii of $10 \mathrm{~nm}$, larger than expected from the spectral overlap of the QD emission and gold localised surface surface plasmon absorption, are obtained.
\end{abstract}

Keywords: Förster resonant energy transfer, nanocrystal quantum dots, Au nanoparticles, time-resolved emission decay, surface plasmons

\section{INTRODUCTION}

Fluorescence quenching by metal nanoparticles (NPs) has been the subject of intense research, with applications, for example, in the area of nano-sensors [1]. Metal nanoparticles with size below 40nm, when put in close proximity to dyes or QDs, act as a radiative quencher over a broad range of wavelengths [2]. Quenching near a metal nanoparticle occurs by means of rapid nonradiative resonance transfer from an excited probe system, such as QDs or dye molecules, to a metal nanoparticle or a roughened metal surface. This transfer is promoted by the strong optical absorption inherent in plasmonic structures [3] and has been theoretically studied by Govorov et al [4] who derived the nonradiative energy transfer rate between a semiconductor QD and a gold nanoparticle due to dipole-dipole coupling. Reports to date have concentrated on the quenching of chromophores by a single metal NP or by a continuous metal plane. It has been reported that energy transfer between two planes of QDs is mediated by dipole-dipole interaction [5]. For a pair of dipoles, the energy transfer rate $k_{E T} \propto d^{-6}$ [6] where $d$ is the separation between donor and acceptor, as described by Förster resonance energy transfer (FRET). For two planes of dipoles, $k_{E T} \propto d^{-4}$, which shows similar distance dependence as the nano-metal surface energy transfer (NSET) model [7] in which a nanoparticle is treated as a surface. However, it is still an open question whether energy transfer from dyes or QDs to Au NPs is best described by a FRET model which considers resonant dipole states of the QDs and Au NPs, or via the interaction with an infinite surface as in the NSET model. In the FRET formalism, the Förtser radius $R_{0}$ depends on the overlap of the resonant states. Further investigations of $R_{0}$ determined from the spectral overlap and as well as the separation and concentraion dependences of the energy transfer efficiency are required.

In this paper we report detailed investigation of the energy transfer from a plane of QDs (diameter $3.6 \mathrm{~nm}$ ) on a plane of $5.5 \mathrm{~nm}$ diameter $\mathrm{Au}$ spheres via characterization of the photoluminescence (PL) quenching and PL lifetime modification. In this case not only the dependence on the QD-metal separation can be investigated but also the dependence on the Au NP concentration. This can provide greater optimization of nano-sensor sensitivity and further insight into the energy transfer mechanism.

\section{EXPERIMENTAL METHODS}

Colloidal Au NPs with an average diameter of $5.5 \mathrm{~nm}$ stabilized by DMAP [8] were deposited first on a polyelectrolyte buffer layer covering a quartz slide. Negatively charged CdTe nanocrystal QDs, stabilized by thioglycolic acid in aqueous solution [9], were deposited in pure monolayers on quartz slides or in bilayer structure on $\mathrm{Au}$ nanoparticles layer. The QDs and $\mathrm{Au}$ nanoparticles acted as the energy donors and energy acceptors respectively. The monolayer and bilayer structrures are deposited using the layer-by-layer (LbL) 
electrostatic assembly technique, further details on the sample preparation with Au NP monolayers can be found in [10]. As can be seen in Fig. 1(a), in the pure monolayers the donors QDs emission is centred at 650nm.

A Shimadzu UV-2401 PC double-beam UV-Vis recording spectrometer was used to measure the absorption spectra of the layer structures. The room temperature photoluminescence (PL) spectra of the samples were recorded with a Perkin-Elmer LS 55 fluorescence spectrometer using an excitation wavelength of $400 \mathrm{~nm}$. The time-resolved PL decays were measured with a PicoQuant Microtime200 time-resolved confocal microscope system with an excitation wavelength of $470 \mathrm{~nm}$ and an average excitation power of $16 \mathrm{nW}$. For the measurements of the PL intensity decay, a broad band-filter with a nominal centre wavelength of $650 \mathrm{~nm}$ is inserted before the detector. The emission signal of the QD monolayers and QD-Au bilayer was recorded by scanning an area of $80 \mu \mathrm{m} \times 80 \mu \mathrm{m}(150 \times 150$ pixels $)$ with a repetition rate of $8 \mathrm{MHz}$ and an integration time of $4 \mathrm{~ms}$ per pixel.

\section{RESULTS AND DISCUSSIONS}

Firstly the spectral properties of the reference QD monolayer and bilayer of QDs and Au NPs will be presented. After which the dependences of the energy transfer efficiency on the separation between the QD and Au nanoparticle monolayers and the Au nanoparticle concentration will be discussed.

\subsection{ET in a Bilayer of QDs and Au nanoparticles}
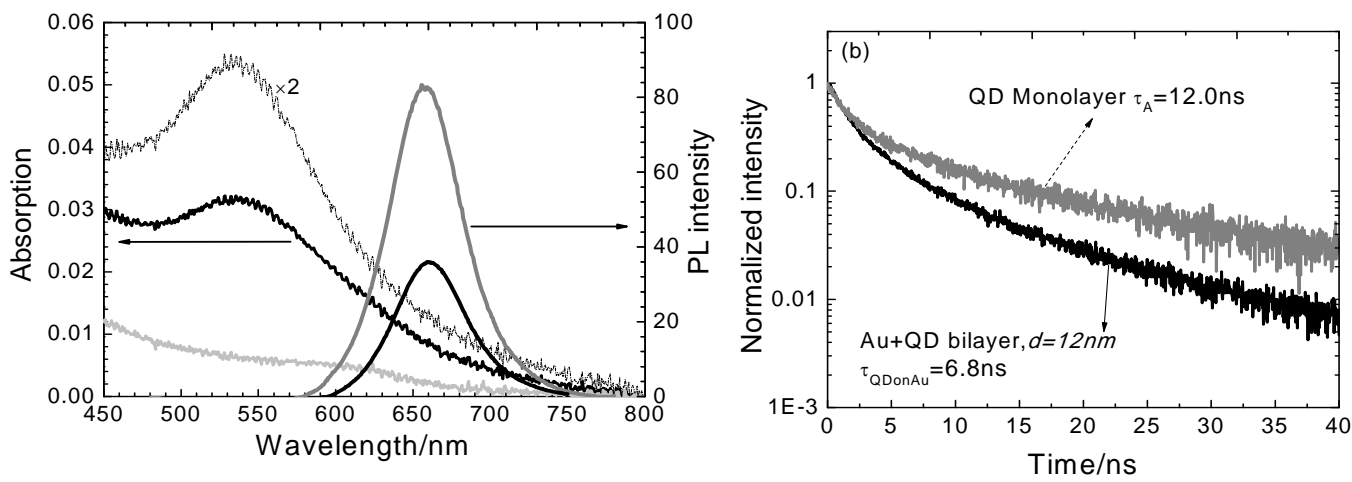

Figure 1. (a) Absorption (right-hand axis) and photoluminescence (PL) spectra (left-hand axis) of Au-QD bilayer structure (black line), QD (grey line) reference monolayer as well as Au monolayer absorption (dots). (b) QD PL decays for the Au-QD bilayer structure (black line) and the reference QD monolayer (grey line).

The absorption and PL spectra for pure QD monolayers as well as bilayer of QDs and Au NPs with the same QD concentration of $c_{Q D}=(1.6 \pm 0.1) \times 10^{17} \mathrm{~m}^{-2}$ are shown in Fig. 1(a). The $\mathrm{Au} \mathrm{NP}$ concentration is $c_{A u}=(0.2 \pm 0.01) \times 10^{17} \mathrm{~m}^{-2}$. The QD size and concentration in the pure monolayers can be extracted from the position of the first absorption peak and the absolute value at this wavelength [11]. The concentrations of the QDs and gold nanoparticles in the bilayer structure were determined by fitting the bilayer absorption spectrum by a weighted sum of the pure QD and gold nanoparticles monolayer absorption spectra [12]. It can be seen that QD emission is quenched by Au NPs by comparison with QD monolayer. Furthermore, normalized PL intensity decays for both structures are shown in Fig. 1(b). The time-dependence of the PL decays $I(t)$ was fitted by a two-exponential decay function: $I(t)=I_{s} \cdot e^{-t / \tau_{s}}+I_{l} \cdot e^{-t / \tau_{l}}+I_{0} \quad I_{0}$ represents the background intensity of the measurement, $I_{s}$ and $I_{l}$ are the two intensity amplitudes and the short and long lifetime component. An intensity-weighted average lifetime $\tau_{\text {avg }}$ is then calculated from the fitting parameters: $\tau_{\text {avg }}=\frac{I_{s} \cdot \tau_{s}^{2}+I_{l} \cdot \tau_{l}^{2}}{I_{s} \cdot \tau_{s}+I_{l} \cdot \tau_{l}}$.

It can be seen that the QD lifetime has been shortened in the bilayer structure. Both PL quenching and lifetime shortening are signatures of energy transfer from the QD layer to the Au NPs layer. The energy transfer efficiency can be determined from the PL quenching, $\frac{I_{Q D}-I_{Q D o n A u}}{I_{Q D}}$, where $I_{Q D}$ and $I_{Q D o n A u}$ represent the QD monolayer emission intensity for the reference sample and the QD emission in the QD-Au bilayer sample. Furthermore, the energy transfer efficiency can also be calculated from the change in lifetime, $\frac{\tau_{Q D}-\tau_{Q D o n A u}}{\tau_{Q D}}$, where $\tau_{Q D}$ and $\tau_{Q D o n A u}$ are the QD PL lifetime for the reference layer and the PL lifetime for the QD-Au bilayer. 
For the example shown in Fig. 1 shows, the PL quenching efficiency is $(39 \pm 6) \%$ in good agreement with the energy transfer efficiency calculated from the change in lifetime, $(43 \pm 4) \%$.

\subsection{Au-QD Distance Dependence and Au Concentration Dependence}

Firstly, the separation dependence of the energy transfer will be discussed. As can be seen from Fig. 2(a), the energy transfer efficiency calculated from both the PL quenching and lifetimes agree within error, both showing decreasing transfer efficiency with increasing separation. The solid line represents a fit of the separation dependence with a model of FRET between two planes:

$$
E_{\text {FRET }}=\frac{1}{1+\frac{2 \cdot R_{e x}^{4}}{\pi \cdot c_{A u} \cdot R_{0}^{6}}}
$$

This includes the centre-to-centre separation $R_{e x}$ that can be determined from the radius of the Au NP (2.75 nm) and the QD $(1.82 \mathrm{~nm})$, the length of the interpenetrating ligand $(0.5 \mathrm{~nm})$ and the spacer layer thickness $\mathrm{d}=(3 \mathrm{to}$ $18 \mathrm{~nm})$ giving $R_{e x}=(\mathrm{d}+2.75+1.82+0.5) \mathrm{nm}$. From fitting with equation (1) a Förster radius, $R_{0}$ can be determined. The best fit for QDs layer on $\mathrm{Au}$ is shown as a solid black line and corresponds to $R_{0}=(10.0 \pm 0.6) \mathrm{nm}$.
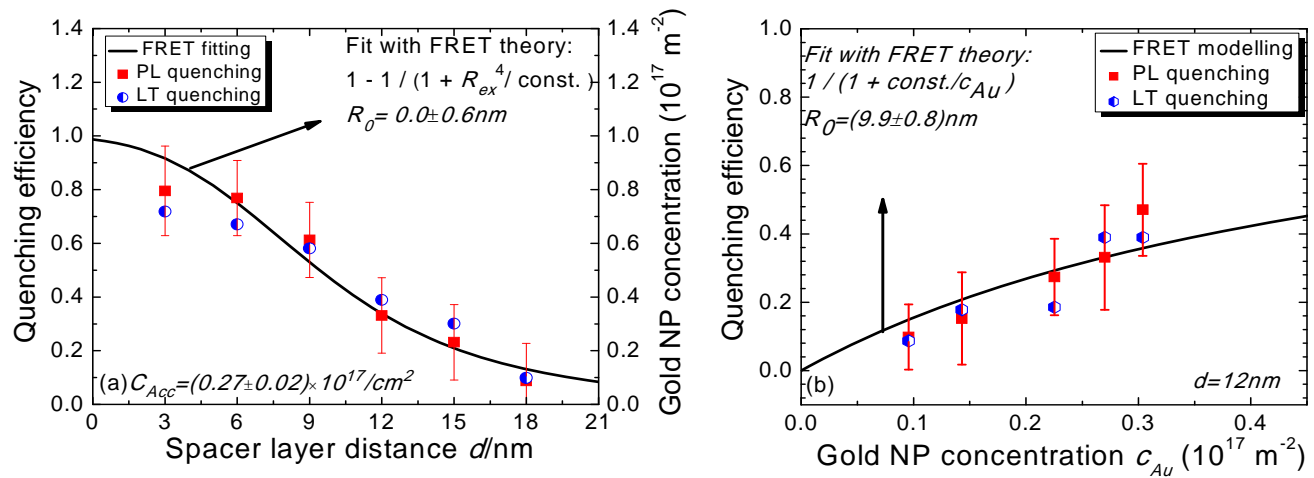

Figure 2. (a) Distance dependence of the energy transfer efficiency determined Au-QD bilayer structures. The solid line represents the distance dependence of the quenching efficiency calculated with equation (1) based on FRET theory. (b) Au concentration dependence of the energy transfer efficiency determined experimentally for QD bilayer structures with a QD-Au layer separation of $12 \mathrm{~nm}$ and calculated with equation (1) (solid line).

In order to further probe the validity of FRET, we also examined the energy transfer efficiency as a function of Au concentration, shown in Fig. 2(b) for spacer layer thickness $d=12 \mathrm{~nm}$. The energy transfer efficiency increases with $\mathrm{Au}$ concentration as seen in the stronger quenching of the QD emission. The trend fits well with FRET theory and $R_{0}=(9.9 \pm 0.8) \mathrm{nm}$ is obtained by fitting with equation (1). This value is in good agreement with that obtained from the separation dependence.

\subsection{Discussion}

Energy transfer efficiency has a $1 / \mathrm{d}^{4}$ dependence for both FRET and NSET models. However, the observed Au concentration dependence of the energy transfer is well described by FRET theory, which indicates a Au nanoparticle, which has comparable size to the QD, could be viewed as a dipole. As noted earlier, in the FRET formalism the energy transfer occurs via resonant states and $R_{0}$ can also be calculated from the spectral overlap of the donor emission and the acceptor absorption. The spectral overlap, $J$, is given by $J=\int_{0}^{\infty} \hat{I}_{D}(\lambda) \cdot \varepsilon_{A c c}(\lambda) \cdot \lambda^{4} d \lambda$, in which $\hat{I}_{D}(\lambda)$ is the area-normalized donor (QD) emission spectrum $\varepsilon_{A c c}(\lambda)$ is the acceptor extinction spectrum, which can be obtained here by scaling the absorption spectrum of the Au NPs with a value of $4.19 \times 10^{6} \mathrm{M}^{-1} \mathrm{~cm}^{-1}$ at the wavelength of the localised surface plasmon absorption peak from the spectra shown in Fig3. The Förtser radius

$$
R_{0}=0.0211 \cdot\left(\frac{\kappa^{2} \cdot Q_{D}}{n^{4}} \cdot J\right)^{\frac{1}{6}}=(5.7 \pm 0.3) \mathrm{nm}
$$


is calculated for a QD monolayer with quantum yield $Q_{D}=6 \%$ and a volume weighted refractive index of the surrounding medium $n=2.1$. As can be seen the $R_{0}$ values obtained from fitting the separation and Au nanoparticle concentration dependence is almost double that calculated from the spectral overlap.

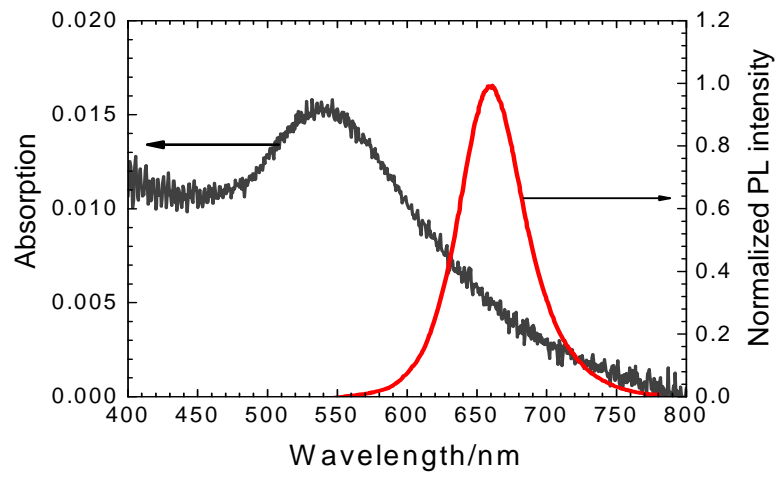

Figure 3 Area-normalized PL of QD (deposited directly on the polyelectrolyte buffer on quartz, without the gold $N P$ layer and Au absorption spectrum with $C_{A u}=0.1 \times 10^{17} \mathrm{~m}^{-2}$ separately.

\section{CONCLUSION}

In summary, PL quenching as well as lifetime shortening are observed indicating energy transfer from a QD monolayer to a Au nanoparticles layer. The energy transfer efficiency is dependent on both the layer separation and the $\mathrm{Au}$ NP concentration. Both dependences could be fitted well with FRET theory which shows a $1 / \mathrm{d}^{4}$ separation dependence and a $1 / \mathrm{C}_{\mathrm{Au}}$ dependence on the $\mathrm{Au} \mathrm{NP}$ concentration. The $R_{0}$ values obtained from separately fitting the separation and Au nanoparticle dependences are in good agreement. This value however is almost double the Förster radius calculated from spectral overlap. This indicates a strong off-resonance interaction between the planes of QDs and Au NP monolayers.

\section{ACKNOWLEDGEMENT}

This work was financially supported by Science Foundation Ireland 10/IN.1/12975.

\section{REFERENCES}

[1] Li X, Qian, et al, Fulorescence quenching of quantum dots by gold nanorods and its application to DNA, Appl. Phys. Lett., vol.94, pp. 063111 - 1-3, Feb, 2009.

[2] Mani Prabha Singh, et al, Involvement of the LSPR Spectral Overlap for Energy Transfer between a Dye and Au Nanoparticle, J. Am. Chem. Soc., pp. 132, 9383-9391, June, 2010.

[3] Maier, S. A. Plasmonics: Fundamentals and Applications (2007).

[4] A. O. Govorov, et al, Theory of plasmon-enhanced Förster energy transfer in optically excited semiconductor and metal nanoparticles, Phy Rev B, vol. 76, pp. 125308-1-16, , Sep. 2007.

[5]Lunz, M, et al, Donor concentration dependence of FRET efficiency in nanocrystal quantum dot structures, Phys. Rev. B vol.83, pp 115423 - 1-10, Mar, 2011.

[6] T. Förster: Zwischenmolekulare energiewanderung und fluoreszenz, Annalen Der Physik 2, 55-57 (1948).

[7] C. S. Yun, et al., Nanometal surface energy transfer in optical rulers, breaking the FRET barrier, J. Am. Chem. Soc. Vol.127, pp. 3115-3119, Feb, 2005.

(8) Gittins, D. I, et al, Spontaneous phase transfffer of nanoparticulate metals from organic to aqueous Media, Chem. Int. Ed. Vol. 40, pp. 3001-3004, Aug. 2001.

[9] S. J. Byrne, et al.: Optimization of the synthesis and modification of CdTe quantum dots for enhanced live cell imaging, J. Mater. Chem., vol. 16, pp. 2896-2902, Jun, 2006; A. L. Rogach, et al.,: Aqueous synthesis of thiol-capped CdTe nanocrystals: State-of-the-art, J. Phys. Chem. C, vol. 111, pp. 14628-14637, Oct. 2007.

[10] Lunz, M., et al, Surface Plasmon Enhanced Energy Transfer between Donor and Acceptor CdTe Nanocrystal Quantum Dot Monolayers, Nano Lett. vol 11, pp. 3341-3345, Aug, 2011.

[11]W. W. Yu, L. H. Qu, W. Z. Guo, and X. G. Peng, "Experimental determination of the extinction coefficient of CdTe, CdSe, and CdS nanocrystals," Chem. Mater, vol. 15, pp. 2854-2860, Jul 2003.

[12]T. Franzl, T. A. Klar, S. Schietinger, A. L. Rogach, and J. Feldmann, Exciton recycling in graded gap nanocrystal structures, Nano Lett, vol. 4, pp. 1599-1603, Sep 2004. 